\title{
ATAXIE TÉLANGIECTASIE ASPECTS GÉNÉTIQUES ET ENZYMATIQUES
}

\author{
Alain AURIAS \\ Institut Curie, 26 rue d'Ulm, 75231 Paris cedex 05
}

L'ataxie télangiectasie (AT) est une maladie récessive autosomique dont la fréquence est estimée à environ 1 cas pour 40 à 100.000 naissances. Il existe par ailleurs de rares syndromes apparentés comme le syndrome de Nijmegen ou l'ataxie Fresno.

L'AT est caractérisée cliniquement par une ataxie cérebelleuse progressive, des télangiectasies cutanéomuqueuses et un déficit immunitaire sévère avec essentiellement baisse des IgA et IgE. Les enfants atteints ont un risque de cancer multiplié par 100 par rapport à leur classe d'âge ( $70 \%$ d'hémopathies et $30 \%$ de carcinomes) et sont extrêmement radiosensibles, ce qui rend souvent délicat le traitement de leurs tumeurs. Ils présentent par ailleurs une cytogénétique très particulière, avec un excès de cassures chromosomiques spontanées et surtout des remaniements très caractéristiques entre les chromosomes 7 et 14 impliquant les gènes de la superfamille des immunoglobulines. La particularité biologique qui est peut-être la plus spécifique est l'absence d'inhibition de synthèse du DNA après irradiation. Cette particularité a permis, par fusion cellulaire, de définir 4 groupes de complémentation différents: AB (55\% des cas), C $(28 \%), \mathrm{D}(14 \%)$ et $\mathrm{E}(3 \%)$, et deux groupes variants $\mathrm{V} 1$ et V2 correspondants au syndromes apparentés (Nijmegen, Fresno, etc...). Cette hétérogénéité génétique fait que la fréquence des hétérozygotes est difficile à estimer de façon précise dans la population générale. Elle pourrait être de l'ordre de 1 à $2 \%$. Cette incidence n'est pas sans importance puisqu'il semble que ces hétérozygotes, bien que ne présentant pas de signes cliniques patents de la maladie, aient un risque accru de faire des cancers: le risque relatif par rapport à la population générale serait de 3,8 pour les hommes et de 3,5 pour les femmes. Pour celles-ci, le risque relatif serait particulièrement important pour le cancer du sein $(x 5,1)$, ce qui suggère que près de $7 \%$ des femmes atteintes de cancer du sein pourraient être hétérozygotes pour un gène AT (revue dans Swift et al., 1991). Il ne semble pas, pour l'instant, que l'on dispose de test simple permettant de repérer avec certitude ces hétérozygotes dans la population générale ou dans des populations à risque (consultation de cancérologie en particulier). 
Les localisations chromosomiques des gènes correspondants aux groupes $\mathrm{AB}, \mathrm{C}$ et $\mathrm{D}$. sont connues. Tous trois sont situés sur le bras long du chromosome 11, au niveau de la bande $11 \mathrm{q} 23$. Les gènes $A B$ et $C y$ ont été assignés par étude de linkage, le gène $D$ par transfert de chromosome 11 ou de fragments de chromosome 11 (revue dans Foroud et al., 1991). Certains arguments font penser que ces différents gènes ne sont pas très proches, les gènes $\mathrm{AB}$ et $\mathrm{C}$ se trouvant clairement localisés entre les locus STMY et D11S132, alors que le gène $D$ serait plus distal que THY1, entre D11S147 et D11S133 (Sobel et al.,1992). Il est à noter qu'un gène candidat pour le groupe $\mathrm{D}$ a été cloné (par restauration partielle d'une radiorésistance en transfectant une séquence cosmidique humaine dans une lignée du groupe D) et que ce gène candidat est effectivement localisé dans la region distale à THY1 (Kapp et al.,1992).

Des publications récentes suggèrent très fortement que les produits des gènes AT normaux seraient impliqués dans le contrôle de l'arrêt du cycle cellulaire en phase G1 (G1 checkpoint), via l'induction de p53 et GADD45 (Kastan et al.,1992). Il est maintenant bien établi que les radiations ionisantes induisent une augmentation de la concentration nucléaire de la protéine p53. Cette augmentation n'est pas observée dans les cellules AT. De manière similaire, les radiations ionisantes induisent rapidement le gène GADD45 dans les cellules normales (et non dans les cellules $\mathrm{AT}$ ), et cette induction nécessite l'existence d'un gène p53 sauvage. $\mathrm{Ce}$ gène contient d'ailleurs une séquence consensus de fixation de la protéine p53. Il est donc vraisemblable que les radiations ionisantes induisent la synthèse de protéine p53, qui, se fixant sur le gène GADD45, induit la synthèse de la protéine correspondante. Les résultats publiés suggèrent très fortement que cette protéine joue un rôle central dans l'arrêt du cycle cellulaire en phase G1 en cas de lésions du DNA. Les cellules AT ayant perdu les possibilités d'induction de p53 et de GADD45, et l'arrêt en G1 en cas d'irradiation, souffrent donc d'un défaut situé en amont de ce système de contrôle du cycle, et l'un des rôles possibles des protéines AT pourrait être d'activer le gène p53 en cas de lésions radio-induites du DNA. 

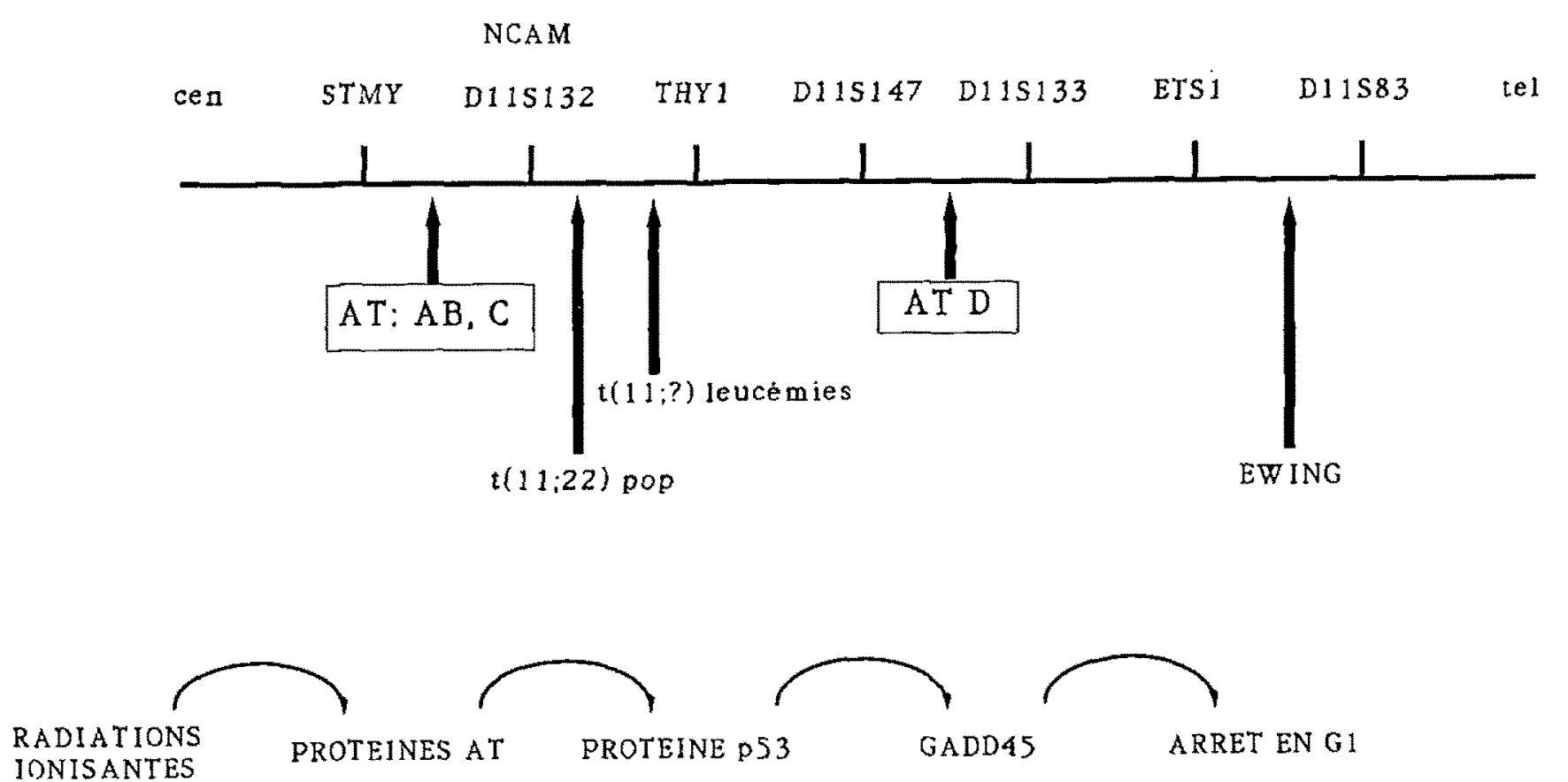

Bibliographie

Swift M., Morrel D., Massey R., Chase C.

Incidence of cancer in 161 families affected by ataxia-telangiectasia New England J Med 1991, 325, 1831-1836

Foroud T., Wei S., Ziv Y., Sobel E., Chao A., Goradia T., Huo Y., Tolun A., Chessa L., Charmley P., Sanal O, Julier C., Concannon P., McConville C., Taylor A., Shiloh Y., Lange K., Gatti R.

Localization of an AT locus to a $3 \mathrm{cM}$ interval on chromosome 11q23: linkage analysis of 111 families by an international consortium

Am J Hum Genet 1991, 49, 1263-1279

Sobel E., Lange E., Jaspers N., Chessa L., Sanal O., Shiloh Y., Taylor A., Weemaes C., Lange K., Gatti R.

Ataxia-Telangiectasia: linkage evidence for genetic heterogeneity Am J Hum Genet 1992, 50, 1343-1348

Kapp L., Painter R., Yu L., van Loon N., Richard C., James M., Cox D., Murnane J

Cloning of a candidate gene for AT group D

Am J Hum Genet 1992, 51, 45-54

Kastan M., Zhan Q., El-Deiry W., Carrier F., Jacks T., Walsh W., Plunkett B., Vogelstein B., Fornace A.

A mammalian cell cycle checkpoint pathway utilizing p53 and GADD45 is defective in Ataxia-Telangiectasia

Cell 1992, 71, 587-597 